\title{
Die eiszeitliche Vergletscherung des Bayerischen Waldes
}

\author{
Von Peter Ergenzinger, Berlin
}

Mit 1 Karte, 2 Abbildungen und 3 Tabellen im Text.

$\mathrm{Zusammenfassung}$. Die Formengemeinschaft der Hochgebiete des Bayerischen Waldes ist oberhalb von etwa $1000 \mathrm{~m}$ Höhe gekennzeichnet durch die Vergesellschaftung von Karen, Steilhängen, steilen Muldentalschlüssen, bandförmigen Hangversteilungen sowie Wasserfällen und Flußschnellen. Diese Formen kommen auch unterhalb der in der Literatur aufgeführten mächtigen Blockmoränen vor und sind Zeugnisse einer weitreichenden Vergletscherung. Die $C^{14}$-Bestimmung einer Probe aus dem Grundmoränenkomplex im Aufschluß bei der Schustersäge im Reschwassertal gab ein Alter von etwa 40800 Jahren.

Es lassen sich drei würmeiszeitliche Stadien unterscheiden: das Maximalstadium, das Blockmoränenstadium und das Karmoränenstadium. Die Schneegrenzen der Stadien betrugen etwa 1060,1140 und $1230 \mathrm{~m}$.

Im Regengebiet bei Zwiesel gibt es Aufschlüsse mit Stauchungserscheinungen in hochgelegenen Terrassen. Diese Vorkommen sind älter als die letzte Eiszeit und werden entsprechend den Befunden im Schwarzwald als Spuren einer noch ausgedehnteren rißeiszeitlichen Vergletscherung gedeutet.

Die Karte der Verbreitung der würmeiszeitlichen Gletscher gibt für die tschechischen Gebiete nur eine extrapolierte Verbreitung an, die auf Grund von Karteninterpretationen entstand und nicht im Gelände überprüft werden konnte.

$\mathrm{S} \mathrm{u} \mathrm{mm}$ a ry. Corries, abrupt slopes, steep heads closing basin-like valleys, waterfalls and rapids are the characteristic association of land forms in the highest parts of the Bayrischer Wald (Bavaria) above about $1000 \mathrm{~m}$. Such forms are also to be found in areas lower than the previously described boulder moraines and offer proof of an extensive glaciation.

A C-14 determination of a sample from the ground moraine occurring in a sandpit near the Schustersäge in the Reschwasser Valley puts the origin of the moraines at about 40,800 years B. P.

The Wurm Glaciation can be divided into three stages: 1, that of the maximum glaciation, 2 , that of the boulder moraines and 3, that of the corrie moraines. The snow lines of the three stages must have been about 1060,1140 and $1230 \mathrm{~m}$. respectively.

Some exposures on high terraces in the area of the R. Regen show signs of glacial pressure effects. These deposits are older than the last glaciation and, like the findings in the Black Forest, indicate an even more widespread Riss Glaciation.

The map shows the maximum extension of the Wurm glaciers in the Bayrischer and the Böhmer-Wald. For the latter area, lying in Czechoslovakia, the evidence has been derived by extrapolation from the Bayrischer Wald and from interpretation of topographical maps without field control.

\section{In ha $1 \mathrm{t}$}

1. Kurzer Uberblick über den Stand der Forschung . . . . . . . . 153

2. Die Formengemeinschaft der Hochgebiete . . . . . . . . . . 153

3. Die glazialen Akkumulationsformen . . . . . . . . . . . 157

a) Die Moränen nahe den höchsten Gipfeln . . . . . . . . 157

b) Die Blockmoränengebiete . . . . . . . . . . . . 158

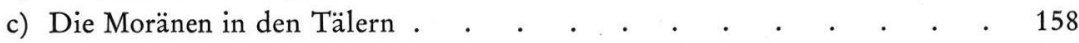

d) Stauchungserscheinungen und Moränenfetzen in hochgelegenen Terrassen 161

4. Die Grübenfelder . . . . . . . . . . . . . . . . 163

5. Die Verbreitung der riß- und würmeiszeitlichen Vergletscherungsspuren und die

Rekonstruktion der klimatischen Schneegrenzen . . . . . . . . 164

Literaturverzeichnis . . . . . . . . . . . . . . . . 166 


\section{Kurzer UUberblick über den Stand der Forschung}

Die Exkursion in den Bayerischen Wald ${ }^{1}$ ) im Anschluß an die DEUQUA-Tagung in Nürnberg 1962 hat gezeigt (vgl. K. KAISER 1963, 227-240), daß noch weithin die von J. Partsch (1882), A. Penck (1882), A. Penck, A. Böhm und A. Rodler (1887) mitgeteilten Ansichten über die Vergletscherung dieses Mittelgebirges gelten. Nach der vorherrschenden Meinung beschränken sich die Spuren der eiszeitlichen Vergletscherung im wesentlichen auf die Kare und das jeweils vorgelagerte Blockmoränenfeld. Die Schneegrenze für diese Vergletscherung wird im Anschluß an A. PencK (1884) mit etwa $1200 \mathrm{~m}$ angegeben (M. BRUSCH 1949, B. FrEnZEL 1959). Die zahlreichen Untersuchungen von A. Rathsburg $(1928,1930,1932-35,1937)$ erweiterten die Zahl der gefundenen Moränen und Kare, weichen aber prinzipiell nicht von der Auffassung von J. PARTSCH und A. Penck ab. Die Ansicht über eine weitergehende Ausdehnung der glazialen Phänomene von F. Bayberger (1886) wurden durch A. Penck, A. Böhm und A. Rodler (1887) widerlegt. Später hat G. PrIEHÄUSSER in zahlreichen Arbeiten (1927, 1930, 1938, 1951 und 1955) zu dokumentieren versucht, daß der Bayerische Wald eine fünfmalige pleistozäne Verfirnung erfahren hat, und daß diese, entgegen der Meinung anderer Autoren (u. a. A. Rathsburg), wesentlich ausgedehnter gewesen sei. Die Kare mit den abschließenden Blockmoränen werden von PRIEHÄUsSER als Rückzugsstadium der letzten Eiszeit angesehen. Während R. von Klebelsberg diesen Befund in sein Handbuch (1949, 658659) mit der Einschränkung aufnimmt, daß die Abtrennung von Spuren älterer Vergletscherungen nicht genügend gesichert erscheine, deuten die meisten Autoren (vgl. u. a. P. Woldstedt 1958, 206) Priehäusser's bis zu Höhen um $480 \mathrm{~m}$ reichende „Firneisgrundschuttdecken " aus 5 Eiszeiten vorwiegend als Solifluktionserscheinungen. Im Folgenden sollen einige Beobachtungen über eine weiterreichende eiszeitliche Vergletscherung des Bayerischen Waldes mitgeteilt werden.

\section{Die Formengemeinschaft der Hochgebiete}

Kennzeichnend für den Bayerischen Wald ist der plötzliche Anstieg aus den ausgedehnten, 700 bis $800 \mathrm{~m}$ hohen Hauptrumpfflächen des Pfahlgebietes zu weitgespannten Rücken und Plateaus mit Höhen von über $1000 \mathrm{~m}$. Im Regen- und Angelgebiet kompliziert sich dieser Bauplan dadurch, daß die Hauptrumpfflächen und die tieferen Niveaus in einzelnen Buchten parallel zur Streichrichtung des Gebirges weit gegen das zentrale Maderplateau vordringen und den Osser- und Arberkamm abtrennen. Im südwestlichen Böhmerwald erreichen die Rücken des Kubany und des Pleckensteins die Höhe des Maderplateaus.

Die großzügige Rumpftreppenanlage, die gekennzeichnet ist durch das Über- und Ineinanderlagern einiger "Formengruppen der Erosion“, wurde durch jüngere Erosionsund Akkumulationsvorgänge mit einem klimatisch bedingten Formenstil überprägt. Im gesamten Böhmerwald tritt in den Hochgebieten eine spezifische, klimabedingte Formengemeinschaft auf. Die Leitform dieser Uberprägung und damit auch dieser Formengemeinschaft sind $\mathrm{K}$ a r e.

1) Das Waldgebirge zwischen dem Pfahlgebiet und den altbesiedelten Becken und weiten Tälern westlich der Linie Pilsen-Budweis wurde bis zum Ende des ersten Weltkrieges Böhmerwald genannt. Als Bayerischer Wald bezeichnete man damals nur das Waldgebirge zwischen dem Pfahl und der Donau. Erst die Abriegelung der Grenzen im Laufe dieses Jahrhunderts führte dazu, den Namen Böhmerwald auf das Waldgebirge jenseits der bayerischen Grenze zu beschränken und die Mittelgebirge westlich der bayerischen Grenze zwischen der Donau und der Further Senke einheitlich als Bayerischen Wald zu bezeichnen. Ich bezeichne im Folgenden als Bayerischen Wald den bayerischen Anteil am Böhmer Wald und als Böhmerwald das gesamte Waldgebirge im hergebrachten Sinne. 
Die bekannten See- und Moorkare wurden zuletzt in der Übersichtskarte von J. Sekrra (1960 und 1961) dargestellt und von P. Ergenzinger (1965) in der Tabelle I zusammengefaßt.

Tabe11e 1

Die Kare des Böhmerwaldes

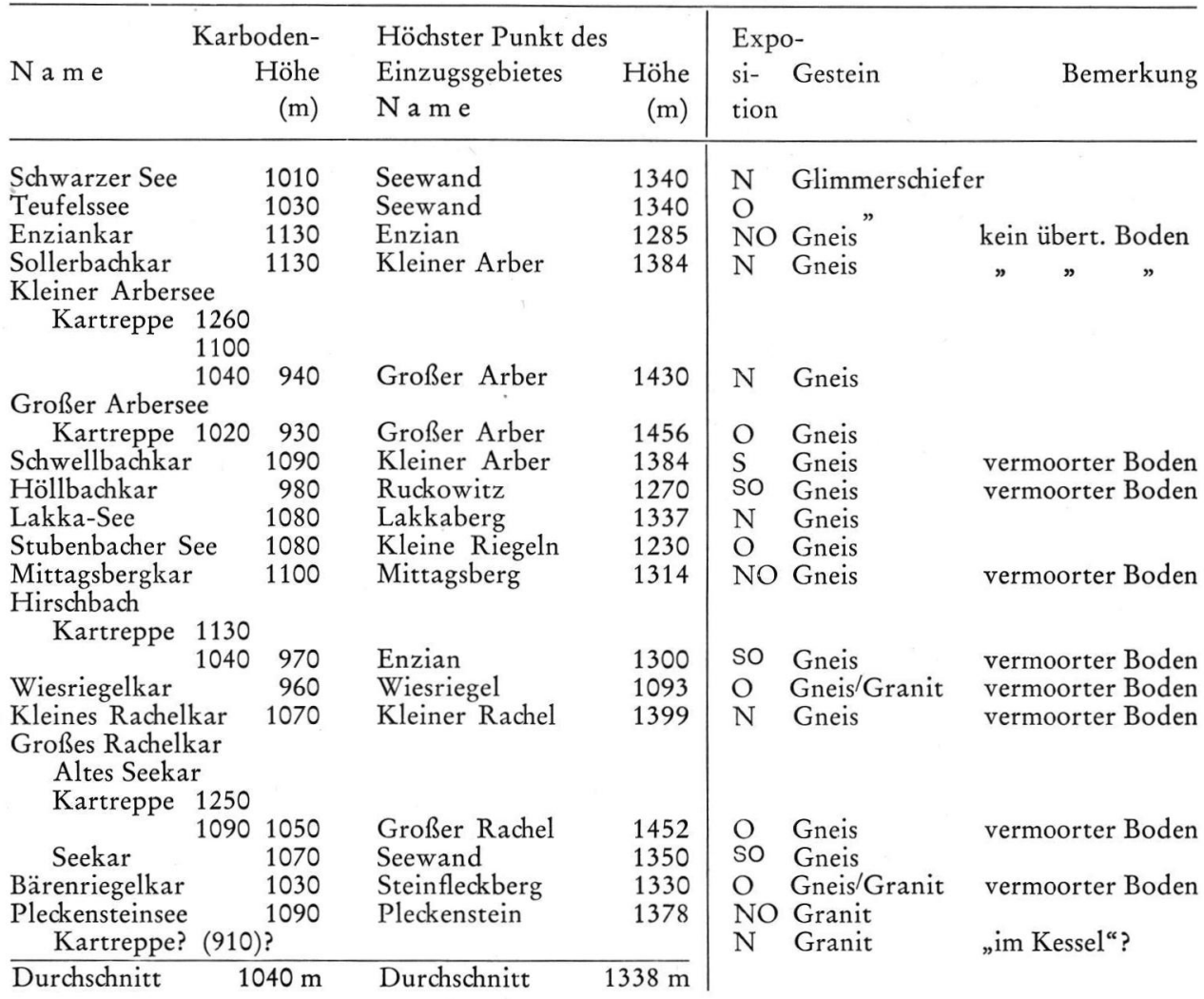

Das arithmetische Mittel der Höhenlage von 18 Karböden ergibt für den Böhmerwald einen Wert von 1040 m. Gemeinsam ist allen bedeutenden Karen eine Exposition nach $\mathrm{N}, \mathrm{NO}, \mathrm{O}$ bzw. SO und die Lage unterhalb von meist über $1300 \mathrm{~m}$ hohen Bergen. Die am besten ausgeprägten und größten Kare wurden im Glimmerschiefer oder in Gneisen ausgebildet, allein das Plöckenstein-Seekar ist im Granit ausgebildet worden. Kennzeichnend für die Kare sind neben den übertieften Karböden die übersteilten Hänge mit Felspartien und Neigungen von über $35^{\circ}$. Die typische Lehnsesselform erreichen annähernd nur die Kare des Schwarz- und des Teufelssees, des Lakka- und des Pleckensteinsees, während z. B. das große Arbersee- und das Rachelseekar jeweils zusammengesetzte Kare (Großkare) sind. Bei den Seekaren ist häufig eine zweigeteilte Karbodenwanne ausgebildet. Am besten ist dies am großen Rachelsee zu beobachten (siehe die Profile bei G. PrieHÄUSSER 1927, Tafel 3). Nach den Lotungen von A. RathSBURG (1928) gilt dies aber auch für den im Grundriß wesentlich einheitlicheren Schwarzsee. Ein erstes Karbecken liegt unter der höchsten Karwand. Das zweite liegt jenseits einer flachen Schwelle am unteren Ende des Karbodens. Die Trennung des Karbodens in ein „Karwandbecken“ und ein „Zungenbecken i. e. S.“ ist aber nur an Karen mit einem über $300 \mathrm{~m}$ hohen Einzugsgebiet zu beobachten. Wie das Kar des Kleinen Arbersees zeigt, kann das Karwandbecken 
auch durch eine Anzahl kleinerer Nebenkarböden ersetzt sein und nur ein „Zungenbecken i. e. S." sich talwärts anschließen. Erreichen die Karhänge keine Höhe von $300 \mathrm{~m}$, so hat das Kar nur einen flach übertieften Karboden. Diesem Kartyp entspricht am besten das kleine Kar am Enzian im Talschluß des Steinbaches.

Talabwärts folgt vor allen Karen des Böhmerwaldes ein Gebiet blockreicher Endmoränen mit Wällen von stellenweise über $20 \mathrm{~m}$ Höhe. Das bekannteste Moränenfeld wird von der Arberseestraße geschnitten. Unterhalb des Rachelsees, des Großen und des Kleinen Arbersees enden diese Blockmassen in etwa 900 m, $850 \mathrm{~m}$ und $790 \mathrm{~m}$ Höhe jeweils nach einer Steilgefällestrecke des Talgrundes.

Am ostexponierten Rand und innerhalb von großen, hochgelegenen Quelltrichtern kommen an vielen Stellen kleine K a roide vor. Sie sind oft nur 50-100 m breit und nur wenige $100 \mathrm{~m}$ lang, haben aber eine sehr scharfe Kante gegen die übrige Mulde und sind bis zu $50 \mathrm{~m}$ gegenüber den umliegenden Hängen eingetieft, Musterbeispiele derartiger Karoide sind am Arberbach (unterhalb P 984), an der Tiefen Seige beim Plattenhäusernriegel (unter $1080 \mathrm{~m}$ ) cder an der Kleinen Flanitz südwestlich der alten Poschinger Jagdhütte (westlich des Kleinen Rachels) in etwa $1050 \mathrm{~m}$ Höhe zu beobachten.

Die Hangneigungen von über $35^{\circ}$, verbunden mit dem Auftreten von Felspartien in Hängen fern des fluvialen Einflußbereichs, beschränken sich nicht auf die Kare und treten, abgesehen von wenigen kleinflächigen, strukturbedingten Vorkommen in tieferen Lagen, im Bereich über $1000 \mathrm{~m}$ Höhe häufig auf. Hervorzuheben sind die Steilh ä nge am Osser (1266 m), Falkenstein (1312 m), Arber (1456 m), Scharzeck (1238 m), Plattenhausenriegel $(1376 \mathrm{~m})$ und Farrenberg $(1203 \mathrm{~m})$. Diese Steilhänge liegen häufig benachbart $\mathrm{zu}$ voll ausgebildeten Karen, weisen jedoch keine O-Exposition, sondern eine W- bzw. meist eine SW-Lage auf. Die aufgezählten Steilhänge sind über Gneisen und Graniten gleichförmig ausgebildet.

Die steilen Karoide sind häufig eingebettet in weite, muldenförmige Tal$s c h l$ üsse. Im Vergleich zu den in Höhen unter $1000 \mathrm{~m}$ im vorderen Bayerischen Wald auftretenden verwandten Formen zeichnen sich die Talschlüsse und Hangmulden der Hochgebiete durch eine besonders steile Umrahmung aus. Häufig verlängert sich der ostexponierte Muldenflügel in einen talabwärts rasch auskeilenden Steilhang mit etwa $30^{\circ}$ Neigung. Während die tieferliegenden Formen nur einen oder mehrere Schwemmschuttfächer im Muldentiefsten haben, liegen im Hochgebiet vor den beiden unteren Enden des Rahmens der Mulden jeweils zwei weitere Schwemmschuttfächer, die den mittleren Schwemmschuttfächer einkeilen. Typisch für diese Konfiguration sind die Verhältnisse z. B. im Sagwassertalschluß zwischen Lusen und Hohem Filzberg, beim Schreyerbach zwischen Plattenhausenriegel und Großem Spitzberg, bei der Flanitz am Kleinen Rachel, beim Hochfall- und Rothbach zwischen Enzian und Kleinem Arber, beim Lohberger Bach am Osser oder beim Pommerbach unterhalb des Kiesrucks.

Diese Formen erinnern an die Quelltrichterkarte nach O. MAULL (1958, 379 f.): „vorwiegend längliche, aber auch fächerartig aufwärts geweitete, steile Böden mit kräftigem oberen Anstieg, aber ohne eigentliche Verflachung".

Außer den weiten muldenförmigen Talschlüssen sind in Hängen mit Einzugsgebieten von etwa $1200 \mathrm{~m}$ auch langgestreckte, steilgeneigte $\mathrm{Muld}$ e $\mathrm{n}$ ä l e r zu finden, die etwa $30-50 \mathrm{~m}$ gegenüber ihrer Umgebung eingetieft sind und an den Seiten von Klippen und Steilhängen begrenzt werden. Unterhalb von etwa $1050 \mathrm{~m}$ Höhe wird der muldenförmige Querschnitt durch einige kegel- und zungenförmige Akkumulationsformen sowie stellenweise auch durch steilhangige kleine Ursprungsmulden gestört. Weder der Scheuereckbach über Spiegelhütte, noch der Bach südlich des Großen Falkensteins, noch der Schwarzbach unterhalb des Hochzellberges bei Bodenmais vermochten diese Akkumulationen zu durchschneiden. Ein gleicher muldenförmiger Talquerschnitt tritt aber andererseits bei wenig Gefälle auch im Grund von größeren Tälern auf. Derartige Talstrecken liegen je- 
weils oberhalb von Sohlentalabschnitten, haben häufig eine Großblockbedeckung und werden von rezentem Bach zumindest oberhalb des Ubergangskegels zwischen Mulden- und Sohlentalabschnitt kräftig zerschnitten. Im Talursprungsgebiet folgen oberhalb des Muldentalabschnittes jeweils Talverengungen mit Steilgefällestrecken. Die besten Beispiele für weitgespannte Muldentäler sind das Teufelsbachtal bei Arberhütte $(710 \mathrm{~m})$ und das Reschwassertal bei der Schustersäge $(820 \mathrm{~m})$ unterhalb von Finsterau.

Im Gebiet der höchsten Erhebungen des Bayerischen Waldes überwiegen jedoch nicht die Karwände und die Steilhänge mit über $35^{\circ}$ Neigungen, sondern neben flachen Talhängen und Ebenen die Hänge und weite muldenförmige Talschlüsse mit Neigungen von $25^{\circ}-30^{\circ}$. Derartige Neigungen treten aber nicht nur in den Hochlagen des Bayerischen Waldes auf, sondern auch am Abfall zur Donau am SW-Rand des vorderen Bayerischen Waldes. In der Höhenregion über ungefähr $1000 \mathrm{~m}$ tragen diese Hänge aber meist eine dichte Grobblockdecke, während die tieferliegenden Steilhänge normalerweise eine Wanderschuttdecke mit vereinzelten Grobblöcken aufweisen. Derartige steile Grobblockhänge sind über grobblockig verwittertem Granit, z. B. am Pleckenstein oder am Plattenhausen, naturgemäß am weitesten verbreitet und stellenweise sogar in ausgedehnten, vegetationslosen Blockmeeren bloßgelegt. Sie fehlen aber auch nicht über Gneis (Südhang, Waldhäuser Riegel, Rachel- und Arberhänge) und treten selbst im Gebiet des Glimmerschiefers am Osser auf.

Im Gebiet der weiten Muldentäler und unterhalb der Kare werden die Hänge stellenweise durch hangabwärts ziehende bandförmige $\mathrm{Hangversteilungen}$ gegliedert. Das eindringlichste Beispiel dafür findet sich über dem Schwarzbach im hinteren Reschwassertal und wird durch den Verlauf des ,Finsterauer Steiges' nachgezeichnet. Die Hangversteilung setzt über den Blockmoränen des ehemaligen Bärenlochgletschers in $1040 \mathrm{~m}$ Höhe an und zieht sich bis zum Reschbach $(980 \mathrm{~m})$ hin. Dort liegt die Versteilung noch immer über $100 \mathrm{~m}$ über dem Talgrund. Am gegenüberliegenden Hang des Hohen Filzberges sind gleichartige Bildungen zu beobachten. Derartige Hangversteilungen sind auch kennzeichnend für andere Täler des hinteren Bayerischen Waldes, wie z. B. im Höllbachtal unter dem Falkenstein, dem Geigenbachtal unter dem Arber, dem Hirschbachtal unter dem Rachel, dem Sagwassertal unter dem Lusen und dem Soller- und Schwellbach unterhalb des Kleinen Arbers. Am besten sind sie in den Seewänden westlich des Kleinen Arbersees ausgeprägt. All diese bandförmigen Hangversteilungen haben eine wesentlich steilere Neigung als das Talgefälle. Sie durchschneiden häufig ältere Verebnungsreste und Hangknicke und sind somit nicht fluviatil bedingt, im allgemeinen auch nicht strukturbedingt.

Neben den steilsten Hängen sind auch die steilsten Talstrecken, die Bach- und Flu ßschnellen, im Bayerischen Wald auf die Bereiche beschränkt, die ein Einzugsgebiet von über $1000 \mathrm{~m}$ Höhe haben. In den gestuften Tallängsprofilen der Bäche und Flüsse treten am Rand des Vorderen Bayerischen Waldes und im Pfahlgebiet Neigungen im Tallängsprofil bis zu maximal 105\% (Große Ohe unterhalb von Spiegelau) auf. Im allgemeinen erreichen die Steilstrecken jedoch höchstens auf kurzen Strecken Neigungen von über $45 \%$. Die einzigen natürlichen $W$ a s se $\mathrm{rfälle} \mathrm{liegen} \mathrm{bis} \mathrm{auf} \mathrm{die} \mathrm{an} \mathrm{Kare}$ geknüpften (z. B. Geigenbachfall über dem Großen Arbersee) an Bächen mit einem um $1300 \mathrm{~m}$ hohen Einzugsgebiet. Sie kommen am Hochfallbach bei Unterried in $800 \mathrm{~m}$ Höhe vor, am Moosbach westlich Bodenmais in $750 \mathrm{~m}$ und $800 \mathrm{~m}$ Höhe, am Riesbach über Bodenmais in 960 und $1000 \mathrm{~m}$ Höhe (Kleinhüttenbach, Wildauer Bach) und am Kleinen Deffernik über Regenhütte in 1000 m Höhe vor. Naturgemäß sind alle diese Wasserfälle strukturell begünstigt, aber ähnliche Strukturen ergeben in tieferen Lagen nur Steilstrecken im Längsprofil und keine. Wasserfälle. Kennzeichnend für die Bäche der Hochgebiete sind neben den vereinzelt auftretenden Wasserfällen der kurze, mehrfache Wechsel von Steilstrecken mit oft über $200 \%$ Gefälle mit anschließenden Flachstrecken. In den Block- 
moränengebieten unterhalb der Kare sind diese Bachschnellen am eindrucksvollsten ausgebildet. Das gleiche Phänomen ist aber auch z. B. im Oberlauf der linken Nebentäler des Weißen Regens oberhalb von Lohberghütte bis herunter in Höhen von 800 bzw. $750 \mathrm{~m}$ (Seebach) zu beobachten und wiederholt sich am Kleinen Deffernik östlich von Lindbergmühle bis in Höhen von $900 \mathrm{~m}$ oder im Geigenbachtal am Pleckenstein. Charakteristisch ist für diese Bachschnellen, daß ihre Lage häufig durch eine Anreicherung von Großblöcken (Kantenlänge über $70 \mathrm{~cm}$ ) bedingt und nur selten an den Ausbiß harter Schichten gebunden ist.

Aus den aufgezählten morphologischen Befunden ergibt sich eine spezielle, klimamorphologisch verursachte Formengemeinschaft der Hochgebiete, die in ihrer Verbreitung gebunden ist an Bereiche mit einem Einzugsgebiet von über $1000 \mathrm{~m}$ Höhe. Die Untergrenze dieser Formengemeinschaft ist umgekehrt proportional zum höchsten Punkt des jeweiligen Einzugsgebietes. Sie sinkt, wenn dieser Berg relativ hoch ist, bis auf Höhen von etwa $700 \mathrm{~m}$. Daraus ist zu schließen, daß der Einfluß der glazialen Formung sich nicht auf die Kare und die Gebiete innerhalb der Blockmoränen beschränkt. Auch außerhalb dieser Gebiete treten, wie G. PriehäUsser stets betonte, glaziale Spuren auf.

\section{Die glazialen Akkumulationsformen}

a) Die Moränen nahe den höchsten Gipfeln.

Bei den Untersuchungen im obersten Reschwasser-Schwarzbachtal ergab sich (P. Ergenzinger 1965, 15-16), daß die Solifluktionsdecke in den Aufschlüssen oberhalb von etwa $1150 \mathrm{~m}$ Höhe fehlt. Zahlreiche Schürfungen am Weg zum Kirchlinger Stand zeigten jeweils unter einem hellbraunen Waldboden ein regelloses Gemenge von kantengerundeten und kantigen Granitblöcken. Selbst bei einer Aufschlußtiefe von 2,4 m waren zwischen hangender und liegender Verwitterungsdecke keine strukturellen Unterschiede festzustellen. Beobachtungen beim Bau der Holzabfuhrstraße Rachelsee-Martinsklause bestätigten auch in diesem Gebiet des Fehlen von Solifluktionserscheinungen über der Grobschuttdecke sowie über den grussandreicheren Schuttdecken. Während am Arber-Lifthang bei der Talstation in $1060 \mathrm{~m}$ Höhe noch eine bis $1,5 \mathrm{~m}$ mächtige Solifluktionsdecke angeschnitten wurde, zeigte der von G. PRIEHÄUSSER (1951) mitgeteilte Aufschlußbefund vom Falkensteingipfel (ca. $1300 \mathrm{~m}$ Höhe) eine „Firneisgrundschuttdecke" über einem gestauchten Grus, der auf keinen Fall als eine Solifluktionsdecke zu interpretieren ist.

Andererseits treten in diesem Höhenbereich Nebenkare und Moränen auf: z. B. das Nebenkar "Geige“ im Großen Arberseekar (1 $170 \mathrm{~m})$, das oberste Nebenkar im Kleinen Arberseekar (1160 m), das Nebenkar unter dem Waldschmidthaus am Rachel mit einer Moräne in $1230 \mathrm{~m}$ Höhe. Hinzu kommen die blockreichen Wallmoränen des obersten Reschbachtales (zwischen 1050 und $1100 \mathrm{~m}$ ) und im obersten Kleinen Schwarzbachtal (um $1200 \mathrm{~m}$ ). Sehr wahrscheinlich entspricht diesen obersten Moränen auch das kleine Rachelwiesenkar mit den bereits von A. RathsBURG (1930) beschriebenen Moränen in $1080 \mathrm{~m}$ Höhe (südlich des Sattels zwischen dem Großen und dem Kleinen Arber).

Für die höchsten Gebiete über etwa $1150 \mathrm{~m}$ Höhe ist neben dem Auftreten von glazialbedingten Formen und Akkumulationen ein Fehlen von periglazialen Abtragungsformen und -massen kennzeichnend. Nach der Aufschlußbeschreibung von R. REICHELT (1964) entsprechen die Verhältnisse am Brocken im Harz über etwa $1050 \mathrm{~m}$ Höhe meinen Befunden in den höchsten Gebieten des Bayerischen Waldes. Auch am Brockenhang tritt eine Moränendecke ohne eine überlagernde Solifluktionsmasse auf.2)

2) Vielleicht bilden die obersten waldfreien Hochflächen und Gipfel (Lusen, Rachel, Arber) im Bayerischen Wald eine Ausnahme dieser Regel. Dort könnte, wenn die Kleinstformen nicht trügen (palsenartige Buckel und Miniatursteinringe auf dem Arberplateau), die Untergrenze der rezenten Solifluktionsstufe nicht mehr fern sein. 
b) Die Blockmoränengebiete.

Aus den Gebieten der mächtigen Blockmoränenfelder wurden bereits mehrfach Aufschlüsse beschrieben. Zur Zeit befinden sich die besten Aufschlüsse an der neuen Rachelstraße unterhalb des Karsees. Wie bereits die Oberflächenformen vermuten lassen, bestehen diese blockreichen Endmoränen überwiegend aus gerundeten Blöcken verschiedenster Größe in lehmiger oder sandiger Matrix. Diese Blockmoränen erreichen unterhalb des Großen Arbersees mit $810 \mathrm{~m}$ ihre tiefste Lage. Von NW nach SW kommen Blockmoränen in den in der Tabelle 2 aufgeführten Tälern des Bayerischen Waldes vor.

$$
\text { Tabelle } 2
$$

B lockmoränen des Bayerischen Waldes

\begin{tabular}{lclr}
\hline Ta l & $\begin{array}{c}\text { Höhenlage der } \\
\text { untersten } \\
\text { Blockmoränen }(\mathrm{m})\end{array}$ & $\begin{array}{l}\text { Höchster Berg } \\
\text { des } \\
\text { Einzugsgebietes }\end{array}$ & (m) \\
\hline Steinbach & 940 & Enzian & 1285 \\
Sollerbach & 850 & Kleiner Arber & 1384 \\
Seebach & 830 & Großer Arber & 1456 \\
Geigenbach & 810 & Großer Arber (Klippe, W) & 1420 \\
Höllbach & 930 & Lackaberg & 1320 \\
Hirschbach & 950 & Enzian & 1300 \\
Kleiner Regen & 850 & Rachel & 1452 \\
Seebach & 910 & Rachel Sroß & 1452 \\
Schreyerbach & 940 & Gusen Spitzberg & 1350 \\
Sägwasser & 980 & Moorberg & 1371 \\
Großer Schwarzbach & 900 & Schwarzberg & 1370 \\
Reschbach & 910 & 1314
\end{tabular}

Im allgemeinen sind die Blockmoränen unterhalb von Karen am mächtigsten und eindrucksvollsten entwickelt, während sie unterhalb von übersteilten Talschlüssen seltener zu Wällen angehäuft sind und oft durch eine einförmige Blockanreicherung im Talgrund vertreten werden (z. B. Schreyerbach bzw. Höllbach). Im Höllbachgespreng treten aber selbst unter Felswänden keine Moränenwälle auf.

c) Die Moränen in den Tälern.

Bei allen tieferen Gletscherlagen fehlt die Anreicherung von Blockschuttmassen zu mächtigen Endmoränenwällen und ganzen Wallfeldern. Die glazial erzeugten Vollformen sind hier oft nur flache Wälle oder Kuppen wie im Reschwassertal, eine glaziale Serie „en miniature" ist nur am Gegenbach unter dem Pleckenstein in $700 \mathrm{~m}$ Höhe zu finden.

Neben den morphologischen Indikatoren liefert der Aufschluß bei der Schustersäge im Reschwassertal (P. Ergenzinger, 1965, 17, Abb. 3 und 6) den besten Hinweis auf eine ausgedehnte Vergletscherung des Bayerischen Waldes. Der Aufschluß liegt in $840 \mathrm{~m}$ Höhe, ist etwa $7 \mathrm{~m}$ tief in den untersten Talhang eingeschnitten und zeigt folgendes Profil:

Mächtigkeit

$$
\text { Profil des Moränenaufschlusses bei der Schustersäge }
$$

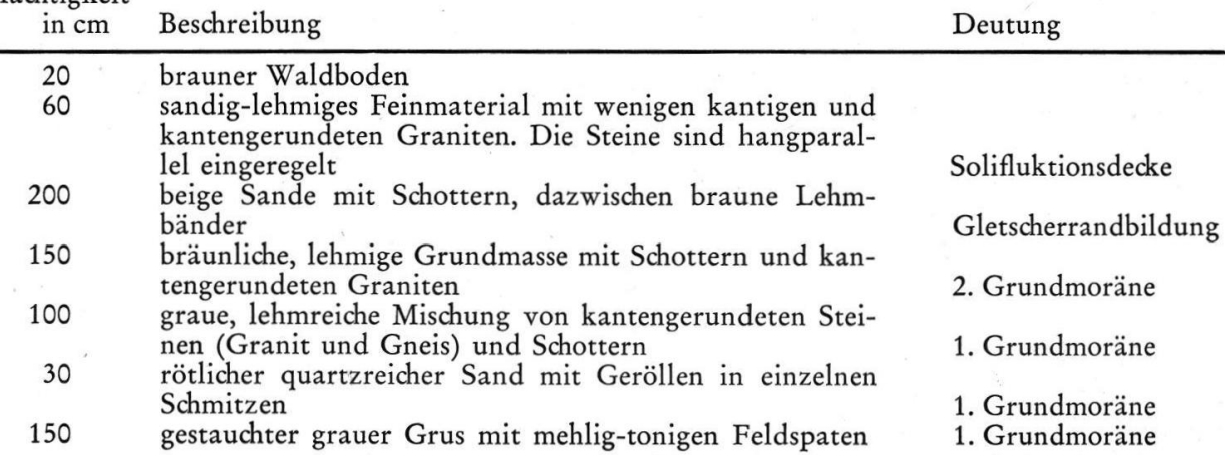


Der unterste Horizont ist gekennzeichnet durch ein Neben-, Uber- und Ineinander von Grusen, Sanden, Lehm und blockreichem Material. Im nördlichen Aufschlußhang liegt ein über 1,5 m langer polierter Granitblock mit Gletscherschrammen. Beide Beobachtungen deuten auf einen ehemaligen mächtigen Gletscher im Bereich der Schustersäge. Die Gruspartien werden überlagert von einer graufarbigen unteren steinreichen Lehmpackung der unteren ersten Grundmoräne. Stellenweise ist diese wiederum in zwei Schuppen gegliedert, zwischen denen rötliche und hellbraune Sande lagern. Die auflagernde obere Lehmpackung besitzt eine bräunliche Farbe und ist etwas sandreicher. Diese zweite Grundmoräne unterscheidet sich nicht nur durch ihre Farbe, sondern auch durch eine vom Liegenden unabhängige Bewegungsstruktur. Zwischen den beiden Grundmoränen liegt kein Verwitterungshorizont. Der Aufschluß zeigt deutlicher als der von Poser \& HövermanN (1951, 86 Abb. 6) aus dem Odertal beschriebene die knetende und stauchende Wirkung des ehemaligen Gletschers. Allerdings sind die gestauchten Akkumulationsmassen nicht so bedeutend wie jene des ehemaligen Titiseegletschers im Joostal.

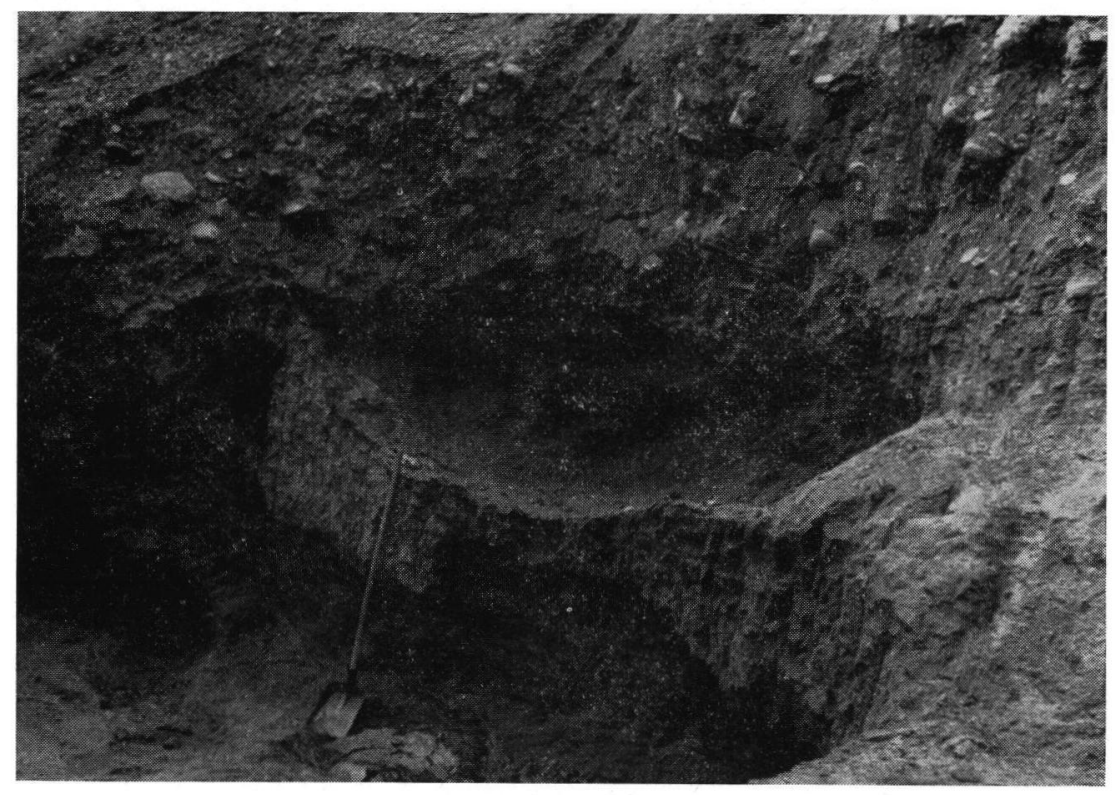

Abb. 1. Glazial gestauchte Grusscholle unter Grundmoräne bei der Schustersäge im Reschwassertal.

Zwei Vorkommen in demselben Tal oberhalb der Schustersäge ergeben auch einen Hinweis für die Mächtigkeit dieses ehemaligen Gletschers. Auf dem Talsporn unter der Ederplatte liegen über vergrustem Granit Gneisblöcke. Am anschließenden Talhang steht nur Granit an, die Blöcke müssen aus dem Gneisgebiet im Oberlauf des Reschbaches durch einen über $75 \mathrm{~m}$ mächtigen Gletscher herantransportiert worden sein. Damit in Zusammenhang steht die bereits erwähnte schräg hangabwärts führende Hangversteilung beim „Finsterauer Steig“. Dieser Gletscher muß, wie der Seitenmoränenwall kurz oberhalb der Mündung des Vorderen Stimmelbaches beweist, im Reschwassertal bis etwa in die Höhe von P 783 m gereicht haben. Damit erklärt sich auch die talaufwärts von P 783 auftretende Anreicherung von meterlangen Granitblöcken und der sprunghafte Beginn einer $2,5 \mathrm{~m}$ hohen Flußterrasse unterhalb von P 783.

Der glaziale Formenschatz im südlichen Rachelvorland wird im Zusammenhang mit den Grübenfeldern noch erörtert werden. Nördlich des Rachels liegt an der Mündung von 
Hirschbach und Kleinem Regen an der Straße zum ehemaligen Forsthaus Hirschbach ein weiterer Moränenaufschluß in $765 \mathrm{~m}$ Höhe. Dort wird ein flacher Rücken angeschnitten, der sich an den Osthang des Hirschbachtales anschmiegt. Unter einem braunen Waldboden mit dünner Humusauflage folgt in den obersten $80 \mathrm{~cm}$ eine Anreicherung von Blöcken und Geröllen in sandigem, schwach lehmigem Grundmaterial über einer $200 \mathrm{~cm}$ mächtigen, steinreichen Grundmoräne. Die Einregelungsmessungen nach der Methode von H. Poser \& J. Hövermann (1951) ergaben ein Moränenspektrum mit einer Dominanz der vertikalen Komponenten $\left.(23,19,25,39) \cdot{ }^{3}\right)$

Im Arbergebiet sind die Talmoränen am besten in der Form von Grundmoränenablagerungen bei Arberhütte zu beobachten. Oberhalb der Mündung in den Großen Regen durchfließt der Teufelsbach eine enge Bachkerbe, die sich bei Arberhütte in einer weiten, flachgemuldeten Talwanne verliert. In der Mitte der Talwanne liegen Herden von meterlangen Gneisblöcken. Talabwärts läßt sich die Talwanne als Terrasse über der Bachkerbe noch mindestens bis in $690 \mathrm{~m}$ Höhe weiterverfolgen.

Wie der Aufschluß am Straßeneinschnitt zwischen P 713 und den Höfen am Bach zeigt, wurden die Gneisblöcke von einem Gletscher herantransportiert. In dem Aufschluß liegt unter $80 \mathrm{~cm}$ mächtigen solifluidal umgelagerten Schottern in sandig-lehmiger Matrix eine über $3 \mathrm{~m}$ mächtige Schicht mit großen Blöcken, Schottern und Kiesen in lehmig-sandiger Grundmasse. In dieser Schicht wurden die über $20 \mathrm{~cm}$ langen Schotter und Blöcke eingemessen. Die Reihe 25, 20, 19, 34 ist die typische Verteilung für eine Moräne, es überwiegen die Steine mit steilgestellten Längsachsen.

Einen hohen Grobblock- und Lehmgehalt hat auch die Schuttmasse, die am Seebach unterhalb der Blockmoränen des Kleinen Arbers durch den Bau der Waldstraße zwischen der Mündung Seebach/Weißer Regen bis unter das Seehäusl in $815 \mathrm{~m} \mathrm{bzw.} 714 \mathrm{~m}$ Höhe jeweils am linken Talhang angeschnitten wurde. Die Einregelungsmessung ergab an beiden Aufschlüssen ein dem Teufelsbach vergleichbares Moränenspektrum (36, 24, 16, 27 bzw. $36,21,15,31)$. Außerdem finden sich oberhalb im Seebachtalgrund zahlreiche verstreute große Blöcke.

Nach den Messungen in 845 und $860 \mathrm{~m}$ Höhe ist auch die Schuttmasse, die bei der Mündung des vorderen Bramersbaches den Talgrund auskleidet, als Grundmoräne zu deuten $(27,22,24,26$ und 24, 14, 20, 42). Im Steinbachtal folgen unter den erwähnten Wallmoränen in $840 \mathrm{~m}$ Höhe weitere Wälle bis nahe P 786. Dort ändert sich gleichzeitig der Talquerschnitt, indem der flache muldenförmige Talboden durch ein Kerbtal abgelöst wird.

In den Tälern südlich des Rückens Schwarzeck-Großer Arber sind bisher bis auf das erwähnte kleine Kar und die Moränen bei der Rachelwiese am Schwellbach keine glazialen Formen erwähnt worden. Am Schwellbach liegen die untersten Endmoränen in der Talweitung bei der Mündung von Arberbach und Schwellbach in $1013 \mathrm{~m}$ Höhe. Unterhalb

3) Die Grundlagen der Einregelungsmessungen wurden erstmals von K. Richter (u. a. 1932, 1936, 1951) und G. Lundquist (1948) an Hand von Beispielen aus ehemaligen Inlandeisgebieten dargelegt. H. Poser \& J. Hövermann führten derartige Messungen im Harz durch. Sie vereinfachten das Verfahren, maßen statt mit dem Kompaß mit einer nach dem Tal- oder Hanggefälle orientierten Meßtafel und faßten die Richtungen zu vier Gruppen zusammen.

Die Längsachsen der 1 . Gruppe sind zwischen $0^{\circ}$ bis $30^{\circ}$ aus der Richtung quer zum Talgefälle abgelenkt worden, die 2. Gruppe umfaßt den Bereich zwischen $30^{\circ}$ bis $60^{\circ}$ und die 3. Gruppe die Gesteine mit einer Einregelung der Längsachsen zwischen $60^{\circ}$ und $90^{\circ}$. In der 4. Gruppe werden alle Gesteine zusammengefaßt, deren Längsachsen gegenüber der Meßfläche eine vertikale $\mathrm{Ab}$ lenkung von über $30^{\circ}$ erfahren haben. Um ein verläßliches Spektrum zu erhalten, werden 100 Steine eingemessen. Die Methode ist mit einfachen Hilfsmitteln rasch durchzuführen. Sie genügt zur einfachen Unterscheidung von fluvialen Ablagerungen (ausgeglichenes Spektrum), solifluidalen Ablagerungen (Maximum in der 1. Gruppe) und glazialen Ablagerungen (hoher Anteil der 4. Gruppe). Für den Bayerischen Wald bot sich diese Methode auch besonders deswegen an, weil auf Grund deı Petrographie Zurundungsmessungen an Gneisgeröllen zu keinen zuverlässigen Werten führen. 
von $1000 \mathrm{~m}$ Höhe finden sich noch glazialverdächtige Hangversteilungen und Wasserfälle. Dies gilt insbesondere für das Riesbachtal, wo sich unterhalb der Wasserfälle eine Hangversteilung am rechten Talhang bis zu der Großblockakkumulation in $715 \mathrm{~m}$ Höhe hinunterzieht. Im Hochfalltal läuft eine markante Hangversteilung in $780 \mathrm{~m}$ Höhe aus. Günstiger sind die Verhältnisse im Tal des Kleinen Deffernik über Regenhütte. Unter der Bachsteilstrecke mit meterhohen kleinen Wasserfällen liegen in einer Talweitung zwischen P 939 und $850 \mathrm{~m}$ Höhe flache, großblockreiche Wälle. Eine Messung in der Schuttmasse am Anschnitt des neuen Holzabfuhrweges oberhalb von P 939 ergab ein für Grundmoränen in hängiger Lage typisches Spektrum: 33, 28, 10, 21.

Im Höllbachtal östlich des Falkensteins liegt ein über $10 \mathrm{~m}^{3}$ großer Block inmitten der Niederterrasse bei P 786. Wegen der mangelhaften Aufschlußverhältnisse ließ es sich nicht entscheiden, ob der Block ansteht oder ob er transportiert wurde. Im benachbarten Tal des Kleinen Deffernik lagert ein nur wenig kleiner Block in der Wiese oberhalb der Bauhüttenbrücke (P 769) jedoch über einem geröllreichen, lehmigen Sand. Da der über $6 \mathrm{~m}^{3}$ große Block weder durch den Bach noch durch einen Bergsturz herbeigeführt werden konnte, muß ein glazialer Transport angenommen werden.

Glazigene Schuttmassen wurden beim Wegebau am Steinschachtenbach (Abb. 1) oberhalb von Buchenau in $980 \mathrm{~m}$ Höhe angeschnitten. In lehmiger, etwa $3 \mathrm{~m}$ mächtiger Pakkung lagern regellos kantengerundete Blöcke, Scherbenschutt und schlecht gerundete Schotter. Die Schuttmasse ist bis zu P $917 \mathrm{zu}$ verfolgen und hat folgendes Moränenspektrum: 31, 22, 14, 27.

Die glazialen Formen des südöstlichen Bayerischen Waldes wurden von mir bereits an anderer Stelle beschrieben (P. Ergenzinger, 1965). Neben den bereits erwähnten Vorkommen im Reschbachtal sind die Moränen im Gegenbachtal unter dem Pleckenstein besonders erwähnenswert.

d) Stauchungserscheinungen und Moränenfetzen in hochgelegenen Terrassen.

Unterhalb der Bereiche mit den beschriebenen Spuren einer Talvergletscherung sind an wenigen Stellen Zeugnisse einer noch weitergehenden Vergletscherung zu finden. Gemeinsam ist diesen Vorkommen ihre Lage in und auf Terrassen.

Durch Bauarbeiten an der Straße Zwiesel-Grafenau in der Kühau an der Kurve bei $\mathrm{km} 94$ entstand in $606 \mathrm{~m}$ Höhe ein wichtiger Aufschluß (Abb. 2). Die Basis des Vorkommens liegt etwa $6 \mathrm{~m}$ über der Niederterrasse in der Höhe des Bahnkörpers und besteht aus vergrustem Granit mit mehlig-tonigen Feldspaten und verwittertem Glimmer. Darüber folgen über $4 \mathrm{~m}$ mächtige gut gerundete Grobschotter mit einer maximalen Länge von $60 \mathrm{~cm}$. Die Schotter lagern sehr fest in rötlichen bis leicht grünlichen Sanden und fetten Lehmen. Entgegen dem fluviatilen Habitus der Gerölle ist ihre Lagerung völlig ungeregelt. Die Einregelungsmessung ergab die Reihe: 12, 16, 24, 49. Der abnorm hohe Wert der vertikal gelagerten Schotter ergibt eine den Meßreihen in Solifluktionsdecken genau spiegelbildliche Verteilung und ist als Grundmoräne zu deuten. Die Zurundung der Schotter und ihre gestauchte Lagerung lassen sich durch die Annahme einer glazialen Überfahrung von fluvialen Schottern deuten.4) Vielleicht gehört in diesen Zusammenhang auch der von G. Priehäusser beschriebene Aufschluß in der Schotterterrasse bei Ludwigstal (1955, Tafel 3, Fig. 2) mit einer „verschobenen Zersatzscholle zwischen Firneisgrundschuttdecken".

4) In einer Unterredung wies Dr. G. PriehäUsSER darauf hin, daß in der „Kühau“ derartige Aufschlüsse schon mehrfach zu beobachten waren (z. B. am Prallhang des Kleinen Regens unterhalb von Dörfl). PriehäUsser deutete diese Vorkommen schon immer als Moränen (vgl. Priehäusser 1955).

11 Eiszeitalter und Gegenwart 


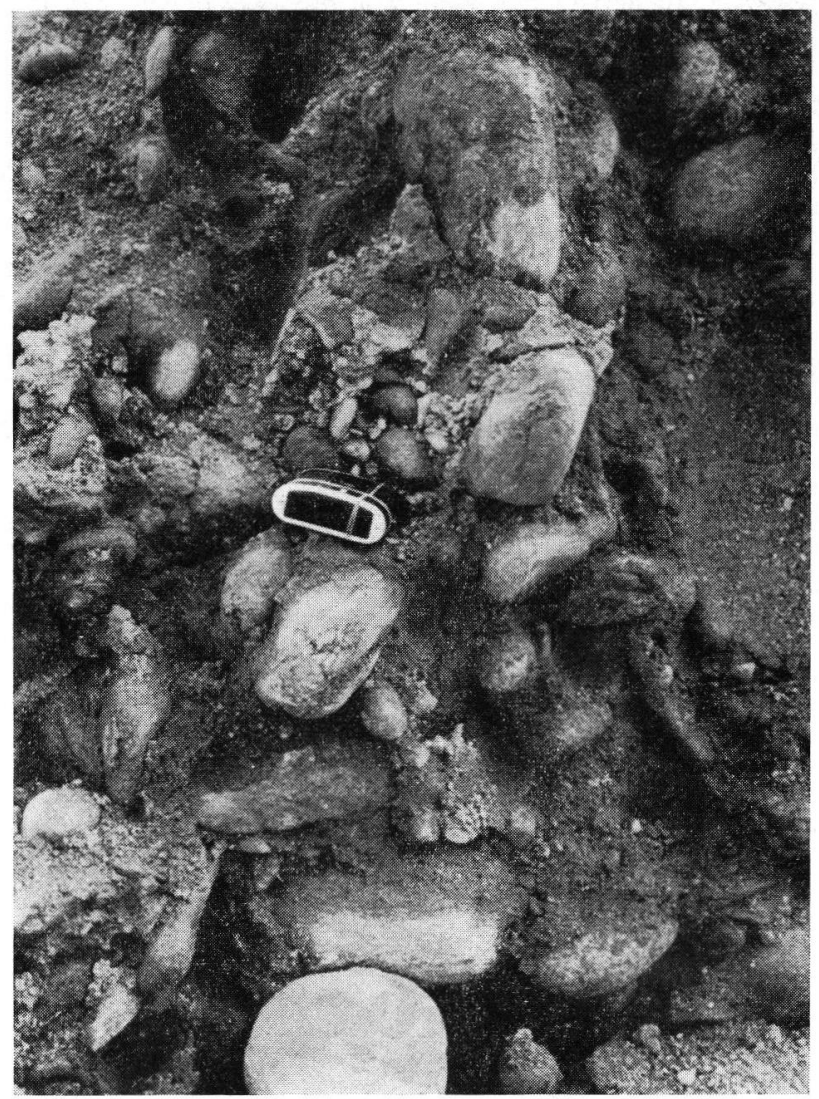

Abb. 2. Straße zwischen Grafenau und „in der Kühau“ (bei km 94; 606 m). Die Aufnahme zeigt in einem Ausschnitt der ca. 1,5 m hohen Aufschlußwand wohlgerundete Gerölle in lehmigem Bindemittel mit wirrer Lagerung.

Im Tal des Großen Höllbaches wird östlich von Lindbergmühle im bis $6 \mathrm{~m}$ hohen Prallhang zwischen der Mündung des Kleinen Höllbaches und der Schwarzen Brücke in etwa $700 \mathrm{~m}$ eine Schuttmasse angeschnitten, die auf Grund der Einregelung (25, 18, 19 ; 25) und der vorwiegend lehmigen Matrix als Grundmoräne anzusprechen ist.

In der „Sandau“ unterhalb des Zwieseler Waldhauses wurzelt ein breiter asymmetrischer Rücken in $660 \mathrm{~m}$ Höhe am Südhang des Drähberges. Der Rücken fällt steil zum Großen Deffernik und flach nach W zum Schmelzbach. Ein Aufschluß liegt mitten auf dem Rücken an der Schneise bei P 630,8 in 643 m Höhe. Unter dem abgeräumten Boden lagert über $3 \mathrm{~m}$ mächtig ein Gemenge von Schottern, Kiesen und wenig gerundeten Blöcken (Kantenlänge bis zu $60 \mathrm{~cm}$ ) in lehmiger Packung. Die Einregelungsmessung mit der Reihe 31, 22, 18, 29 und die Form des Rückens weisen auf eine Deutung als Endmoräne hin.

Aus diesen wenigen Befunden folgt, daß unterhalb der dem Talgrund oder der Niederterrasse auflagernden glazialen Akkumulationen weitere Grundmoränenreste auf der ersten Terrasse über der Niederterrasse auftreten. Auf Grund dieser Beobachtungen im Zwieseler Gebiet bin ich geneigt, die untersten Moränenrücken im Reschwassertal gegenüber meinen früheren Ausführungen (ERGENZINGER 1965, 18/19) anders zu datieren. Auch diese Vorkommen liegen über der Niederterrasse und stammen daher wohl ebenso wie die Vorkommen bei Zwiesel aus der nächstälteren Vergletscherungsphase. Nach diesen 
Befunden einer sehr weitreichenden Vergletscherung ist es auch möglich, den über $5 \mathrm{~m}$ hohen Rücken auf der Saußwasserterrasse bei P 694 für eine weitere derartige Moränenbildung zu halten. Ebenso könnten die tiefliegenden, glazialverdächtigen Formen am Windischbach in $820 \mathrm{~m}$ Höhe zu dieser Gruppe gehören.

\section{Die Grübenfelder}

Als erster untersuchte G. Priehäusser (1927 und 1938) die „Grübenfelder" und deutete alle Vorkommen als Vorzeitformen, die unter dem Einfluß von Gletschern norwegischen Typs $(1938,107)$ beim Abschmelzen von Toteis entstanden sind $(1938,110)$. Die Grübenfelder bestehen aus einem engräumigen Nebeneinander von Hügeln, Rücken, Wannen, Trichtern und schmalen kerb- oder sohlenförmigen Tälchen. Die Voll- und Hohlformen erreichen eine Reliefenergie von weniger als $10 \mathrm{~m}$; das größte Grübenfeld ist knapp $300 \mathrm{~m}$ lang und etwa $70 \mathrm{~m}$ breit und liegt in der „Rannenau“ östlich von Lindbergmühle. Alle Grübenfelder liegen auf Schotterfluren, zumeist in Talweitungen.

PRIEHÄUSSER (1938) veröffentlichte für die folgenden Vorkommen Kroquisaufnahmen im Maßstab $1: 7000$ :

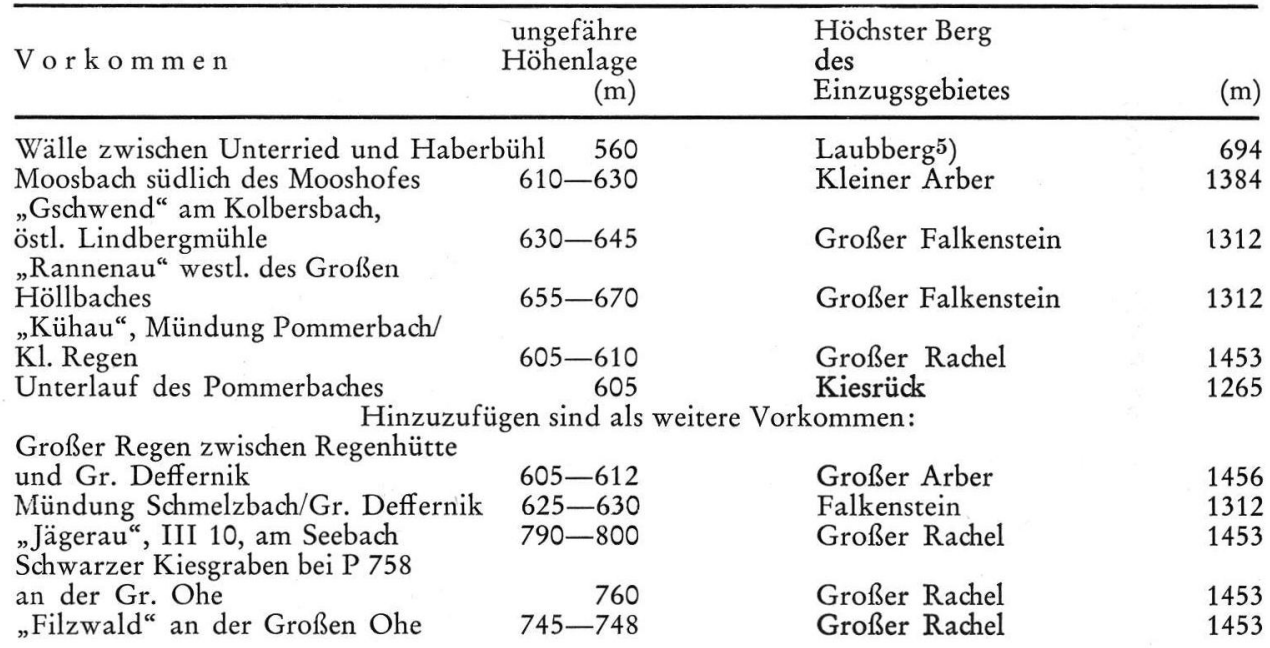

Wie diese Aufstellung zeigt, ist eine Abhängigkeit zwischen der Höhe des Einzugsgebietes und der Höhenlage der Grübenfelder nicht festzustellen. Insbesondere widersprechen die Rücken und Wälle bei Haberbühl-Unterried einer derartigen Relation. Gemeinsam ist allen Vorkommen die Lage in Gneisschotterfluren und die Lage im Niveau der Niederterrassen. Außerdem häufen sich die Rinnen und Hohlformen stets am Rand zu höher gelegenen Akkumulationsterrassen und reichen auch nie unter das Grundwasserniveau. Wegen der Lage im Niederterrassenniveau und der scharfen Ausprägung der Kleinformen ist eine präwürmeiszeitliche Anlage für alle diese Kleinformen auszuschließen.

Die von G. Priehäusser (1938, 98-100) für die Grübenfelder postulierte Abfolge von 4 Grundmoränen mit 3 zwischengeschalteten Schotter- bzw. Sandlagen ist in einem Aufschluß wohl kaum zu finden. Ein räumlicher Zusammenhang zwischen Grundmoränenablagerung und Grübenfeldbildung ist nach meinen Untersuchungen nur im südlichen

5) Bei der Annahme einer Transfluenz von Eis über den niederen $\mathrm{Paß}$ oberhalb von Unterried ist der $1285 \mathrm{~m}$ hohe Enzian der höchste Gipfel des Einzugsgebietes. 
Rachelvorland (P. Ergenzinger 1965, 13-14, Abb. 2) zu beobachten. Im obersten Grïbenfeld (Jagen III 10, $\mathrm{r}=03280 \mathrm{~h}=24160$ ) ist folgende Schichtenfolge aufgeschlossen:

$110 \mathrm{~cm}$ lockere, wohlgerundete Schotter mit wenig sandigem Zwischenmittel (Bachschotter)

$300 \mathrm{~cm}$ Gerölle und Blöcke mit Schottern in verfestigtem Lehm mit wenig Sand (Grundmoräne).

In der Umgebung des Aufschlusses treten bis zu 2,5 m hohe drumlinartige Rücken, bis zu 5 m hohe Wälle, abflußlose Hohlformen und geradlinige Kerbtälchen auf.

Überall, wo die Grübenfelder aber aus Kerb- und Sohlentälchen und dazu benachbart liegenden Rücken bestehen, ist allein nach der Form eine anthropogene Bildung derartiger Grübenfelder nicht auszuschließen. Stellenweise drängt sich der Eindruck geradezu auf, daß die Grübenfelder durch einen selektiven Abbau der Niederterrasse über dem Grundwasserniveau erst jüngst entstanden (Moosbach, „Gschwend“, „Kühau“).

Es ergibt sich für die Entstehung der Grübenfelder nur im südlichen Rachelvorland ein räumlicher Zusammenhang mit Grundmoränenvorkommen. Die übrigen Vorkommen sind vielleicht eher als Folgeerscheinung der Quarzsuche der Glashütten zu deuten. ${ }^{6}$ ) Ein Beweis für eine tiefreichende Vergletscherung ist allein aus der Vergesellschaftung von auffälligen Kleinformen in den Grübenfeldern nicht abzuleiten.

\section{Die Verbreitung der riß- und würmeiszeitlichen Vergletscherungsspuren und die Rekonstruktion der klimatischen Schneegrenzen}

Die Spuren der ehemaligen eiszeitlichen Vergletscherungen des Bayerischen Waldes ordnen sich in einfaches Schema. Alle Vorkommen, die im Zusammenhang mit der Niederterrasse stehen, zeugen für die würmeiszeitliche Vergletscherung, die Vorkommen auf höheren Terrassen sind als Spuren der rißeiszeitlichen Vergletscherung zu deuten.

Das würmeiszeitliche Alter der Talmoränen oberhalb der Moränenfetzen in den hochgelegenen Terrassen bestätigte die C14-Datierung von Holzresten aus der Grundmoräne bei der Schustersäge. Die Holzreste entstammen einer gestauchten, millimeterdicken Lage der oberen Grundmoräne und befanden sich $2 \mathrm{~m}$ unter der Oberfläche. Die Datierung am Natuurkundig Laboratorium der Rijks-Universiteit te Groningen (Gr. N. -4406) ergab ein Alter von $40800+4400-2800$ B. P. und muß „wegen der Möglichkeit von Verunreinigung ... als ein Mindestalter angesehen werden ..." ${ }^{\text {" }) ~ D i e ~ w u ̈ r m e i s z e i t l i c h e n ~ T a l-~}$ moränen entstammen nach dieser Bestimmung aus dem Hochwürm.

Im Areal oberhalb der Karmoränen fehlt die Solifluktionsdecke. Die jüngste solifluidale Überformung fand wahrscheinlich während der jüngeren Tundrenzeit statt. Für die Karmoränen ist somit eine Entstehung während der jüngeren Tundrenzeit anzunehmen. Die Blockmoränen sind demnach in die Spätphase der letzten Eiszeit einzuordnen.

Die in Tabelle I durchgeführte Mittelung der Karbodenhöhen des Böhmerwaldes gibt einen ersten Hinweis auf die Höhe der maximalen letzteiszeitlichen Vergletscherung. Um die klimatische Schneegrenze für kleinere ehemalige „Gletscher mit geringem Höhenunterschied zwischen dem untersten Gletscherende und dem höchsten Punkte des Einzugsgebietes" zu ermitteln, gibt es nach H. Lours (1954/55, 416) die Möglichkeit, zwischen den beiden Extremwerten zu mitteln. Da die Höhenunterschiede zwischen diesen Punkten im Böhmer Wald maximal nur etwa $700 \mathrm{~m}$ erreichen und auch die längsten würmeiszeitlichen

6) Die Untersuchungen von Manske und STERnberg (1965) über die von PrieHäUSSER ebenfalls glazial gedeuteten Grübenfelder im Oberpfälzer Wald ergeben auf Grund von Urkunden, Holzkohlefunden und durch die Vergesellschaftung mit Staudämmen und Zuleitungsgräben eine Deutung der Grübenfelder als Seifenhügel des spätmittelalterlichen Erztagebaus.

7) Brief vom 31. August 1965 - C 14/98-65. 
Gletscher des Bayerischen Waldes (Reschwasser- und Hirschbachgletscher) nur etwa $7 \mathrm{~km}$ lang waren, ist diese einfache Methode anwendbar. Ihr Ergebnis ist dem Mittel aus der gemittelten Firnfeldumrahmung und dem Gletscherende vorzuziehen, weil sich der Wert für die Firnfeldumrahmung für die verschiedenen Gletscherstände ändert und die resultierenden Schneegrenzen somit dann nicht mehr voll untereinander vergleichbar sind. Der gegen die einfache Mittelung zwischen dem höchsten Punkt des Einzugsgebietes und dem Gletscherende vielfach erhobene Einwand, daß ein überragender hoher Berg das Ergebnis verzerrt, trifft für die Mittelgebirge nicht zu. Außerdem ergeben sich auch bei der Mittelung der Firnfeldumrahmung entsprechend große Abweichungen, wenn unter einem allgemeinen Gipfelniveau extrem tiefe, ehemals vielleicht sogar vergletscherte Pässe auftreten.

\section{Tabelle 3}

Berechnung der Schneegrenze für die würmeiszeitlichen Gletscherstadien des Bayerischen Waldes mit Hilfe der Mittelung zwischen dem höchsten Berg des Einzugsgebietes und der jeweiligen Höhenlage der Moränen.

\begin{tabular}{|c|c|c|c|c|c|c|c|c|}
\hline \multirow[b]{2}{*}{$\mathrm{T}$ a 1} & \multirow{2}{*}{\multicolumn{2}{|c|}{ Höchster Berg }} & \multicolumn{2}{|c|}{ Höhenlage der } & \multirow[b]{2}{*}{ 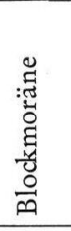 } & \multirow[b]{2}{*}{ 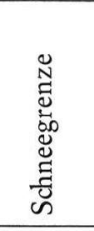 } & \multirow[b]{2}{*}{ 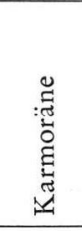 } & \multirow[b]{2}{*}{ 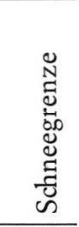 } \\
\hline & & & 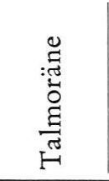 & 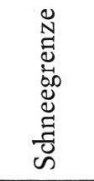 & & & & \\
\hline Geigenbach & Gr. Arber & 1456 & 670 & 1063 & 810 & 1138 & 990 & 1223 \\
\hline Teufelsbach & Gr. Arber & 1456 & 690 & 1073 & & & & \\
\hline Seebach & Gr. Arber & 1456 & 714 & 1085 & 830 & 1143 & 960 & 1208 \\
\hline Sollerbach & Kl. Arber & 1384 & 710 & 1047 & 900 & 1142 & & \\
\hline Steinbach & Enzian & 1285 & 786 & 1035 & 940 & 1112 & 1030 & 1207 \\
\hline Bramersbach & Enzian & 1285 & 845 & 1065 & & & & \\
\hline Hochfallbach & Enzian & 1285 & $800 ?$ & 1042 & & & & \\
\hline Moosbach & Kl. Arber & 1384 & $700 ?$ & 1042 & & & & \\
\hline Rieslochbach & Gr. Arber & 1456 & 695 & 1072 & & & 1020 & 1238 \\
\hline $\begin{array}{l}\text { Kl. Deffernik } \\
\text { (Regenhütte) }\end{array}$ & Hochg'fichtet & 1330 & 825 & 1078 & & & & \\
\hline Höllbach & Lackaberg (imW) & 1320 & 786 & 1053 & 930 & 1125 & & \\
\hline $\begin{array}{l}\text { Kl. Deffernik } \\
\text { (östl. Lindbergmü }\end{array}$ & $\begin{array}{l}\text { Lackaberg } \\
\text { ihle) }\end{array}$ & 1337 & 740 & 1038 & & & 1080 & 1209 \\
\hline $\begin{array}{l}\text { Kl. Höllbach } \\
\text { Steinschachten- }\end{array}$ & Gr. Falkenstein & 1315 & 760 & 1038 & 950 & 1133 & & \\
\hline bach & Kiesrück & 1265 & 850 & 1058 & 975 & 1120 & & \\
\hline Hirschbach & Enzianriegel & 1304 & 760 & 1032 & 960 & 1132 & 1110 & 1202 \\
\hline $\begin{array}{l}\text { Kl. Regen } \\
\text { Große Ohe/ }\end{array}$ & Gr. Rachel & 1453 & 760 & 1107 & 850 & 1151 & 1010 & 1202 \\
\hline Seebach & Gr. Rachel & 1453 & 745 & 1099 & 910 & 1182 & 993 & 1223 \\
\hline Kl. Ohe & Lusen & 1372 & 780 & 1076 & 950 & 1130 & & \\
\hline Sagwasser & Lusen & 1372 & 760 & 1066 & 900 & 1136 & 1160 & 1266 \\
\hline Reschwasser & Moorberg & 1370 & 783 & 1077 & & & & \\
\hline Gr.Schwarzbach & Moorberg & 1370 & & & 900 & 1135 & 1200 & 1285 \\
\hline K1.Schwarzbach & Moorberg & 1370 & & & & & 1210 & 1290 \\
\hline Reschbach & Schwarzberg & 1314 & & & 910 & 1112 & & \\
\hline Teufelsbach & Postberg & 1307 & $883 ?$ & 1095 & & & & \\
\hline Windischbach & Sülzberg & 1130 & 980 & 1055 & & & & \\
\hline Schimmelbach & Hochkamm & 1330 & 730 & 1030 & & & & \\
\hline Gegenbach & Pleckenstein & 1376 & 690 & 1033 & & & & \\
\hline Summe der Reihe & & & & 25459 & & 15891 & & 13553 \\
\hline Arithm. Mittel de & er Reihen: & & & 1065 & & 1135 & & 1232 \\
\hline
\end{tabular}


Wie Tabelle 3 zeigt, ergibt sich aus der Mittelung zwischen dem höchsten Punkt des Einzugsgebietes und der Höhe der untersten würmeiszeitlichen Moränen für den Bayerischen Wald eine Tiefstlage der würmeiszeitlichen Schneegrenze von ungefähr $1060 \mathrm{~m}$ Höhe. Bedeutende Vorstöße erfolgten während des Blockmoränenstadiums bei einer Schneegrenze von ungefähr 1140 m Höhe und im Karmoränenstadium bei einer Schneegrenze von etwa $1230 \mathrm{~m}$ Höhe.

Die wenigen rißeiszeitlichen Vergletscherungsspuren erlauben nur eine grobe Annäherung der Schneegrenze. Bei der Anwendung derselben Methode wie für die würmeiszeitlichen Gletscherstände ergeben sich für die Fundpunkte folgende Werte:

\begin{tabular}{lllrr} 
Höhe der Moräne & $\mathrm{m}$ & Höchster Berg des Einzugsgebietes m & Schneegrenze \\
\hline Sandau, Zwies. Waldh. & 640 & Großer Rachel & 1452 & 1026 \\
Kühau & 600 & Lackaberg & 1337 & 996
\end{tabular}

Auf Grund der Lage und Form des Gebirges sind die ermittelten Schneegrenzwerte des Bayerischen Waldes am besten mit den entsprechenden Werten des Schwarzwaldes zu vergleichen. Pfannenstiel \& Rahm (1963) nehmen für den Südschwarzwald ohne genauere Begründung eine Schneegrenze von $750-800 \mathrm{~m}$ an, die aber „eher in der Nähe von 800 m" liegt $(1963,45)$. Eine Schneegrenzhöhe von $800 \mathrm{~m}$ ergab sich nach REICHELT (1960, 98 und 118) im Hotzenwald. Dieser Wert ändert sich auf Grund der jüngsten Untersuchungen nach ReICHELT $(1966,117)$ zumindest für den östlichen Schwarzwald nicht, obwohl in der Abhandlung von Pfannenstiel \& Rahm eine Schneegrenze von $700 \mathrm{~m}$ vertreten wird. Die würmeiszeitliche Schneegrenze sank nach ERB (1948) bis auf $950 \mathrm{~m}$ im Südschwarzwald $a b$ und lag im Vergleich zu dem entsprechenden Wert des Bayerischen Waldes (1060) um $110 \mathrm{~m}$ tiefer. Die rißeiszeitlichen Schneegrenzwerte differieren aber um maximal $300 \mathrm{~m}$ (1000 m : $700 \mathrm{~m})$ oder minimal um $150 \mathrm{~m}(950 \mathrm{~m}: 800 \mathrm{~m})$.

Wenn man für die Alpen die alten Schneegrenzwerte von A. PencK \& Brückner (1909, 492) zugrunde legt (würmeiszeitliche Schneegrenze $1200 \mathrm{~m}$, rißeiszeitliche Schneegrenze $1100 \mathrm{~m}$ ), so besteht gegenüber den entsprechenden Schneegrenzwerten des Bayerischen Waldes jeweils eine Differenz von etwa $150 \mathrm{~m}$.

Ein Vergleich der süddeutschen Schneegrenzhöhen der Würm- und Rißvergletscherung:

$\begin{array}{ll}\text { Schwarzwald } & \text { B a yerischer Wald } \\ \text { Riß: } \quad 800 \mathrm{~m} & \text { Riß: }>1000 \mathrm{~m} \\ \text { Würm: } 950 \mathrm{~m} & \text { Würm: } 1060 \mathrm{~m}\end{array}$

Alpen-Nordrand

Riß: $\quad 1100 \mathrm{~m}$

Würm: $1200 \mathrm{~m}$

$\mathrm{Ob}$ diese Differenzen das Ergebnis unterschiedlicher klimatischer Bedingungen, oder aber nur den unterschiedlichen Stand der speziellen Erforschung spiegeln, ist noch nicht abzusehen.

\section{Literaturverzeichnis}

Bayberger, F.: Geographisch-geologische Studien aus dem Böhmerwalde. Die Spuren alter Gletscher, die Seen und die Täler des Böhmerwaldes. Peterm. Mitt., Erg.-Bd. XVIII, 81, 1-63, Gotha 1896.

Brusch, M.: Die Höhenlage der heutigen und der eiszeitlichen Schneegrenze in Europa, Vorderasien und den angrenzenden Gebieten. Diss. Göttingen 1948.

ERB, L.: Die Geologie des Feldberges. In: K. MülLER: Der Feldberg im Schwarzwald, 22-96. Freiburg/Br. 1948.

Ergenzinger, P. J.: Morphologische Untersuchungen im Einzugsgebiet der Ilz (Bayerischer Wald). Berliner geogr. Abh., 2, 1-48, Berlin 1965. 
Frenzel, B.: Die Vegetations- und Landschaftszonen Nord-Eurasiens während der letzten Eiszeit und während der postglazialen Wärmezeit. I. Akad. Wiss. Lit. Mainz, Abh. Mathem.Naturwiss. Kl., Jg. 1959, 13, 290-453, Wiesbaden 1960.

KaIser, K.: Die 11. Tagung der Deutschen Quartärvereinigung in Nürnberg vom 21.-27. Sept. 1962. Eiszeitalter u. Gegenw., 14, 227-240, OOhringen 1963.

KLebelsberg, R. vON: Handbuch der Gletscherkunde und Glazialgeologie. II. Wien 1949.

Manske, D. \& Sternberg, H.-G.: Uber einige Grübenfelder im Oberpfälzer Wald. Beobachtungen an sogenannten Toteisbildungen. Mitt. fränk.-geograph. Ges., 11/12, 400-414. Erlangen 1965.

Maull, O.: Allgemeine Geomorphologie. 2. Aufl., Wien 1958.

Partsch, J.: Die Gletscher der Vorzeit in den Karpathen und den Mittelgebirgen Deutschlands nach fremden und eigenen Beobachtungen. Breslau 1882.

Penck, A.: Die Vergletscherung der deutschen Alpen. Leipzig 1882. - - Geographische Wirkungen der Eiszeit. Verh. 4. Dt. Geographent. 66-84, München 1884.

Penck, A., BöHm, A. \& Rodler, A.: Bericht über eine gemeinsame Exkursion in den Böhmerwald. Z. deutsch. geol. Ges., 39, 68-77, Hannover 1887.

Penck, A. \& Brǘckner, E.: Die Alpen im Eiszeitalter. 3 Bde., Leipzig 1901-1909.

Pfannenstiel, M. \& Rahm, G.: Die Vergletscherung des Wutachtales während der Rißeiszeit. Ber. naturf. Ges., 53, 5-61, Freiburg i. Br. 1963. - - Die Vergletscherung des Wehratales und der Wiesetäler während der Rißeiszeit. Ber. naturf. Ges., 54, 209-278, Freiburg i. Br. 1964.

Poser, H. \& Hövermann, J.: Untersuchungen zur pleistozänen Harzvergletscherung. Abh. braunschw. wiss. Ges. 3, 61-115, Braunschweig 1951.

Prienäusser, G.: Der Bayerische Wald im Eiszeitalter. I. Glaziale Spuren in der Umgebung des Großen Arbersees. Geogr. Jh., 40, 133-150, München 1927. - - Die Eiszeit im Bayerischen Wald. Abh. geol. Landesuntersuchg. bayer. Oberbergamt, 2, München 1930. - - Eiszeitliche Toteisbildungen im Bayerischen Wald. Zs. Gletscherkde, 26, 97-111, Berlin 1938. - Der Nachweis der Eiszeitwirkungen im Bayerischen Wald mit Hilfe von Schuttausbildungen. Geol. Bl. NO-Bayern, 1, 81-91, Erlangen 1951. - - Störungen im Zersatz kristalliner Gesteine unter eiszeitlichen Schuttdecken im Bayerischen Wald. Geol. Bl. NOBayern 5, 97-109, Erlangen 1955.

Rathsburg, A.: Die Gletscher des Böhmerwaldes zur Eiszeit. 22. Ber. naturwiss. Ges., 65-161, Chemnitz 1928. - - Neue Beiträge zur Vergletscherung des Böhmerwaldes während der Eiszeit. Mitt. Ver. Erdk., 1929, 65-161, Dresden 1930. - - Die Gletscher der Eiszeit in den höheren deutschen Mittelgebirgen. Firgenwald, 5, 5-25, 65-77, 103-113; 6, 96-112, 126-127; 7, 35-42, 77-107, 148-158; 8, 67-84, Reichenberg 1932-1935. - - Stand und Aufgaben der Eiszeitforschung in den deutschen Mittelgebirgen. Zs. Erdk., 5, 721-734, Breslau 1937.

Reichelt, G.: Zur Frage pleistozäner Vergletscherung im Harz und Thüringer Wald. Erdk., 18, 62-65, Bonn 1964. - - Neuere Beiträge zur Kenntnis der Vergletscherung im Schwarzwald und den angrenzenden Gebieten. Schr. Ver. f. Gesch. u. Naturgesch. der Baar und der angrenzenden Landesteile in Donaueschingen, 26, 108-122. Donaueschingen 1966.

SEKYRA, J.: Působeni mrazu na pudů Kryopedologie se zvlaštnim zřetelem k ČSR. Geotechnica, 27, 1-164, Prag 1960. - - Frostactions on the ground with special reference to Czechoslovakia. Publ. Acad. Sc. Tschécoslovaquie, Prag 1960. - - La carte périglaciaire du Massif Bohémien. Biul. Perygl,- 10, 43-52, Lodz 1961.

Woldstedt, P.: Das Eiszeitalter. 2. Bd., 2. Aufl., Stuttgart 1958.

Manuskr. eingeg. 11. 2. 1967.

Anschrift des Verf.: Dr. Peter Ergenzinger, 1 Berlin 41, Grunewaldstr. 35, II. Geogr. Institut der Freien Universität. 
$\square$ Maximalausdehnung der würm eiszeitlichen Vergletscherung

Kar (Karbodenhöhe unter Nr.3 in der Tabelle I

Caroid

Glazial überformter Quelltrichter

$\because \quad$ Steilhängiges Muldental

Glazial geformte Hangleiste

$0^{0^{000}} \quad$ Rißeiszeitliche Moräne

- Rißeiszeitliche Grundmoräne

.0* Würmeiszeitliche Talmoräne

Würmeiszeitliche Blockmoräne

Würmeiszeitliches Blockmoränenfeld

Würmeiszeitliche Karmoräne

Würmeiszeitliches Karmoränenfeld

$+\quad$ Wichtige Aufschlüsse

至 Grübenfeld

m.M.m Staatsgrenzen

Legende zu der beigehefteten Karte. 